\title{
Visible photoluminescence from planar amorphous silicon nitride microcavities
}

\author{
A. Serpengüzel \\ Department of Physics, Bilkent University, Bilkent, Ankara 06533, Turkey \\ A. Aydinli* and A. Bek* \\ Department of Electrical and Computer Engineering, University of California, Santa Barbara, \\ Santa Barbara, California 93106 \\ M. Güre \\ Department of Physics, Bilkent University, Bilkent, Ankara 06533 Turkey
}

Received April 20, 1998; revised manuscript received July 21, 1998

\begin{abstract}
Fabry-Perot microcavities were used for the enhancement and inhibition of photoluminescence (PL) in a hydrogenated amorphous silicon nitride $\left(a-\mathrm{SiN}_{x}: \mathrm{H}\right)$ microcavity fabricated with and without ammonia. A planar microcavity was realized that included a metallic back mirror and an $a-\mathrm{SiN}_{x}: \mathrm{H}$-air or a metallic front mirror. The PL extends from the red part of the spectrum to the near infrared for the samples grown without ammonia. The PL is in the blue-green part of the spectrum for the samples grown with ammonia. The PL amplitude is enhanced and the PL linewidth is reduced with respect to those in bulk $a$ - $\mathrm{SiN}_{x}: \mathrm{H}$. The numerically calculated transmittance, reflectance, and absorbance spectra agree well with the experimentally measured spectra. (C) 1998 Optical Society of America [S0740-3224(98)00211-2]
\end{abstract}

OCIS codes: $230.5750,250.5230,310.0310$.

\section{INTRODUCTION}

Interest in silicon as a material for optoelectronics has increased recently. With modern processing techniques it will be possible to integrate lasers, photodetectors, and waveguides into optoelectronic Si motherboards to route and modulate optical signals within such $\mathrm{Si}$ motherboards. Silicon oxide $\left(\mathrm{SiO}_{2}\right)$ and silicon oxinitride ( $\left.\mathrm{SiON}\right)$ have both been used in Mach-Zehnder interferometers. ${ }^{1}$ Hydrogenated amorphous silicon ( $a-\mathrm{Si}: \mathrm{H})$ has already been used for planar waveguides. ${ }^{2}$

One advantage of $a-\mathrm{Si}: \mathrm{H}$ is that it can be deposited by plasma-enhanced chemical vapor deposition (PECVD) onto almost any substrate at temperatures below $500 \mathrm{~K}$, which makes it compatible with microelectronic technology. Another advantage of $a-\mathrm{Si}: \mathrm{H}$ (Ref. 3) and of porous silicon ${ }^{4}$ is that they attract interest as potential optical gain media because of their room-temperature visible electroluminescence (EL) and photoluminescence (PL). Visible EL has already been obtained from a pnheterojunction $a$-Si:H-porous silicon optoelectronic device. $^{5}$

Because of their unique optical properties, microcavities and more recently photonic bandgap materials continue to attract attention. ${ }^{6}$ In a microcavity, two electromagnetic and quantum electrodynamic effects occur. First, the microcavity acts as an optical resonator for light rays with specific wavelengths. These wavelengths correspond to the optical modes of the microcavity. Second, in a microcavity the photon densities of states are enhanced at the cavity resonances (optical modes) compared with the continuum of photon states of a bulk sample. The spontaneous emission (luminescence) cross sections at the microcavity resonances are larger than the bulk spontaneous-emission cross sections because of the enhanced photon density of states. ${ }^{7}$ Also, the luminescence cross sections between the microcavity resonances are smaller than the bulk spontaneous-emission cross sections. Alteration (i.e., enhancement and inhibition) of spontaneous emission in planar microcavities has been both observed experimentally ${ }^{8}$ and calculated theoretically. ${ }^{9}$

These properties of the microcavities are used in resonant-cavity-enhanced (RCE) photonic devices, which are wavelength selective and ideal for wavelengthdivision multiplexing applications such as RCE photodiodes, ${ }^{10}$ RCE light-emitting diodes, ${ }^{11}$ vertical cavity surface emitting lasers, ${ }^{12}$ and microdisk $^{13}$ and microwire ${ }^{14}$ lasers.

Planar microcavity effects on the PL of porous silicon ${ }^{15}$ as well as of $\mathrm{Si} / \mathrm{SiO}_{x}$ superlattices ${ }^{16}$ have already been reported. Recently we observed visible PL from $a$-Si:H as well as from its oxides $\left(a-\mathrm{SiO}_{x}: \mathrm{H}\right)$ and nitrides $\left(a-\mathrm{SiN}_{x}: \mathrm{H}\right)$ grown by low-temperature PECVD. ${ }^{17,18}$ In this paper we report, for the first time to our knowledge, the alteration of PL in an $a-\mathrm{SiN}_{x}: \mathrm{H}$ Fabry-Perot microcavity fabricated with ammonia $\left(\mathrm{NH}_{3}\right)$ together with silane $\left(\mathrm{SiH}_{4}\right)$ and nitrogen $\left(\mathrm{N}_{2}\right)$. For the $a-\mathrm{SiN}_{x}: \mathrm{H}$ grown without $\mathrm{NH}_{3}$ the PL extends from the red to the nearinfrared parts of the optical spectrum. However, for $a-\mathrm{SiN}_{x}: \mathrm{H}$ grown with $\mathrm{NH}_{3}$ the $\mathrm{PL}$ is in the blue-green 
part of the optical spectrum. ${ }^{19}$ Although the exact mechanism of the occurrence of PL in bulk $a-\mathrm{SiN}_{x}: \mathrm{H}$ is still under discussion, we have suggested ${ }^{19}$ the use of a quantum-confinement model. ${ }^{20,21}$ It was proposed that our samples should consist of small $a$-Si clusters in a matrix of $a-\mathrm{SiN}_{x}: \mathrm{H}$. The regions with $\mathrm{Si}-\mathrm{H}$ and $\mathrm{Si}-\mathrm{N}$, which have larger energy gaps because of the strong $\mathrm{Si}-\mathrm{H}$ and $\mathrm{Si}-\mathrm{N}$ bonds, isolate these $a$-Si clusters and form barrier regions around them. The PL originates from the $a$-Si clusters.

We intend to conduct experiments, such as current voltage, current optical power, and EL measurements, to study the electrical and optoelectronic properties of our $a-\mathrm{SiN}_{x}: \mathrm{H}$ Fabry-Perot microcavities.

\section{EXPERIMENTAL SETUP}

The room-temperature reflectance and transmittance measurements were made at $0^{\circ}$ with respect to the surface normal and with a spectral resolution of $2 \mathrm{~nm}$. The room-temperature PL spectra were measured with a spectral resolution of $0.1 \mathrm{~nm}$. The PL spectra were then corrected for the responsivity of the spectrometer and the photomultiplier tube. $\mathrm{An} \mathrm{Ar}^{+}$laser with a wavelength of $514.5 \mathrm{~nm}$ (or $457.9 \mathrm{~nm}$ ) and a power of $420 \mathrm{~mW}$ was focused with a $15-\mathrm{cm}$ focal-length cylindrical lens upon samples grown without (or with) $\mathrm{NH}_{3}$. The PL spectra were taken at $0 \pm 3.6^{\circ}$ with the excitation laser at $30^{\circ}$ with respect to the surface normal. During the PL measurements the temperature of the sample was not controlled, and there could be local heating owing to the poor thermal conductivity of the substrates. Local heating reduces the PL efficiency and broadens the PL linewidth. ${ }^{22}$ However, the local heating did not seriously affect the general shape and features of the PL spectra. As we show, in the PL spectra, even though there might have been local heating, we observed strong PL from the samples, and the PL was enhanced by the Fabry-Perot resonances.

\section{HYDROGENATED AMORPHOUS SILICON NITRIDE MICROCAVITY FABRICATED WITHOUT AMMONIA}

The microcavity fabricated without $\mathrm{NH}_{3}$ had a Au back mirror and an $a$ - $\mathrm{SiN}_{x}: \mathrm{H}$-air interface front mirror. First we coated the thin glass substrates with a $100 \mathrm{~nm}$ of $\mathrm{Au}$ to fabricate the high-reflectance back mirror. Then a thin layer of $a-\mathrm{SiN}_{x}: \mathrm{H}$ was deposited onto the Au-coated substrates by PECVD. The flow rate of the PECVD gas $\left(2 \% \mathrm{SiH}_{4}\right.$ in $\mathrm{N}_{2}$ ) was 180 SCCM (SCCM denotes cubic centimeters per minute at STP), the radio frequency power was $10 \mathrm{~W}$, and the pressure in the deposition chamber was 1 Torr. The metric thickness $(L)$ of the $a-\mathrm{SiN}_{x}: \mathrm{H}$ layer grown without $\mathrm{NH}_{3}$ was $1400 \pm 100 \mathrm{~nm}$.

A. Transmittance, Reflectance, and Absorbance of $a-\mathrm{SiN}_{\mathrm{x}}: \mathrm{H}$ Microcavity Fabricated without Ammonia Figures 1(a), 1(b), and 1(c), respectively, show the measured and calculated transmittance, reflectance, and absorbance spectra of the $a-\mathrm{SiN}_{x}: \mathrm{H}$ microcavity fabricated without $\mathrm{NH}_{3}$. We obtained the absorbance spectrum by
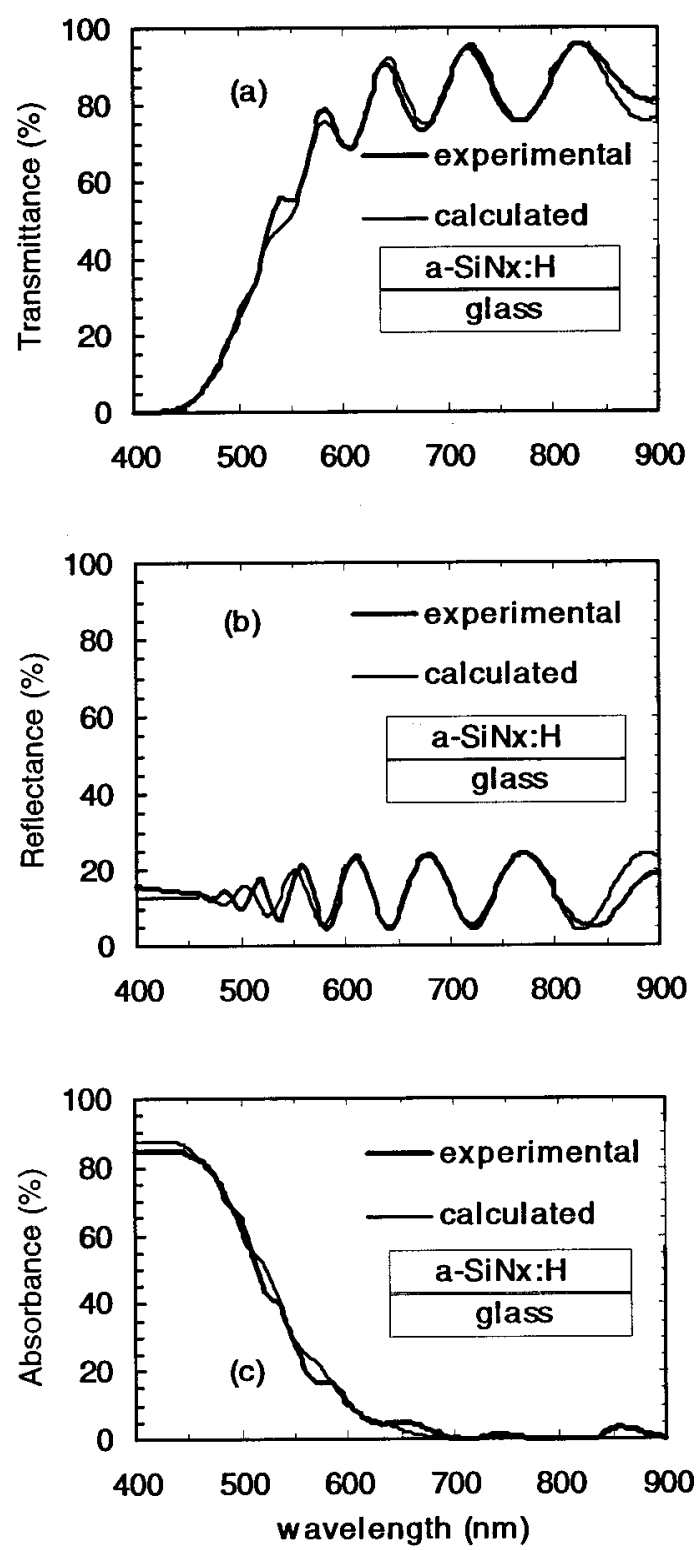

Fig. 1. Experimental and theoretical transmittance, reflectance, and absorbance spectra of an $a-\mathrm{SiN}_{x}: \mathrm{H}$ microcavity fabricated without $\mathrm{NH}_{3}$.

subtracting both the reflectance and the transmittance spectra from unity, i.e., $100 \%$.

In the calculations the total transmitted and the reflected electric fields for each wavelength were obtained with an absorbing Fabry-Perot microcavity model. In this model we assumed the sample to be a onedimensional absorbing dielectric slab. We obtained the experimentally measured absorbance spectrum by subtracting both the experimentally measured transmittance and the reflectance spectra from $100 \%$. This experimentally measured absorbance spectrum was then incorporated into the calculations of the transmitted and reflected electric fields. The calculated transmittance and reflectance at each wavelength were obtained from their respective electric fields. Later, as a final check, the calculated transmittance and reflectance intensities were subtracted from $100 \%$ to yield the calculated absorbance. 
The theoretical fit to the experimental spectra was extremely good. The metric thickness of the $a-\mathrm{SiN}_{x}: \mathrm{H}$ was $L=1376 \mathrm{~nm}$ (which agrees well with the experimental thickness of $1400 \pm 100 \mathrm{~nm}$ ), and the refractive index was $n=2.1$. Both the transmittance (maxima) and reflectance (minima) spectra showed Fabry-Perot resonances at wavelengths $\left(\lambda_{m}\right)$, satisfying the microcavity resonance condition

$$
\lambda_{m}=\frac{2 L n}{m},
$$

where $m$ is the quantized mode number, $L$ is the metric thickness, and $n$ is the refractive index of the microcavity.

The mode numbers of these resonances were found to range from $m=11\left(\lambda_{11}=545 \mathrm{~nm}\right)$ to $m=7\left(\lambda_{7}=835\right.$ $\mathrm{nm})$. The resonances had linewidths of $\Delta \lambda=35 \mathrm{~nm}$ and quality factors of $Q=20$. Below $600 \mathrm{~nm}$ the resonances started to wash out because of absorption of the $a-\mathrm{SiN}_{x}: \mathrm{H}$. The loading of the resonances by the $a-\mathrm{SiN}_{x}: \mathrm{H}$ absorption stopped above $600 \mathrm{~nm}$.

\section{B. Photoluminescence of $a-\mathrm{SiN}_{x}: \mathrm{H}$ Microcavity} Fabricated without Ammonia

Figure 2(a) shows the PL of the $a-\mathrm{SiN}_{x}: \mathrm{H}$ microcavity fabricated without $\mathrm{NH}_{3}$ whose transmittance, reflectance, and absorbance spectra were shown in Fig. 1 . The PL intensity was modulated by the weak Fabry-Perot resonances, which correlate well with the maxima of the transmittance spectrum of Fig. 1(a) and the minima of the
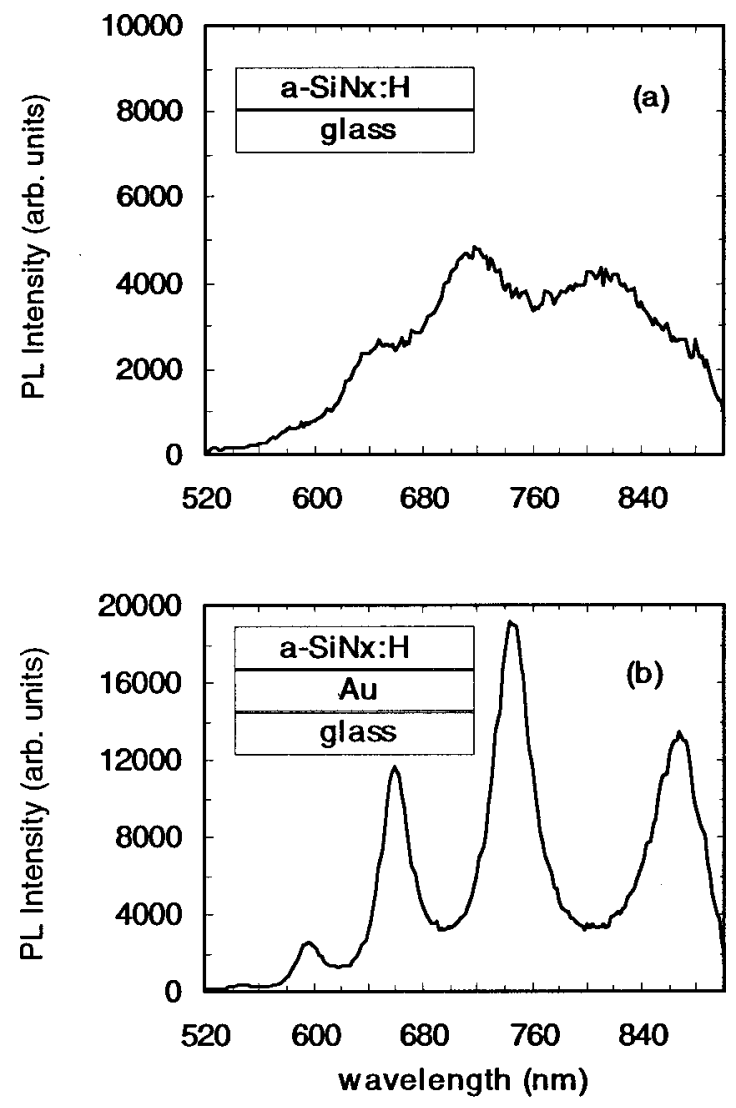

Fig. 2. PL spectra of an $a-\mathrm{SiN}_{x}: \mathrm{H}$ microcavity fabricated without $\mathrm{NH}_{3}$ (a) without the Au back mirror and (b) with the Au back mirror. reflectance spectrum of Fig. 1(b). The red-near-infrared PL had a broad linewidth $(240 \mathrm{~nm})$, which shows that $a-\mathrm{SiN}_{x}: \mathrm{H}$ is a novel photonic gain medium.

Figure 2(b) depicts the PL of the $a-\mathrm{SiN}_{x}: \mathrm{H}$ microcavity with the Au back mirror. The Fabry-Perot resonances had experimental and theoretical linewidths of $\Delta \lambda$ $=25 \mathrm{~nm}$ and quality factors of $Q=30$. The metric thickness of the $a-\mathrm{SiN}_{x}: \mathrm{H}$ microcavity with the $\mathrm{Au}$ mirror was $L=1438 \mathrm{~nm}$ (which agrees well with the experimental thickness of $1400 \pm 100 \mathrm{~nm}$ ) and a refractive index $n=2.1$. The PL was modulated by the strong FabryPerot resonances. The mode numbers of these resonances ranged from $m=11\left(\lambda_{11}=558 \mathrm{~nm}\right)$ to $m=7$ $\left(\lambda_{7}=874 \mathrm{~nm}\right)$.

The PL spectra of Fig. 2 were obtained under the same experimental conditions. Comparing the two spectra in Fig. 2 shows that the PL of the microcavity with the Au mirror has three noteworthy features with respect to the $\mathrm{PL}$ of the $a-\mathrm{SiN}_{x}: \mathrm{H}: \quad$ (1) there is a $2 \times$ increase in the overall spectrum average (i.e., averaging out the FabryPerot resonances), (2) there is a $4 \times$ enhancement of the PL peaks, and (3) the PL dips have similar amplitudes.

The $2 \times$ increase is due to the round trip of the excitation $\mathrm{Ar}^{+}$laser in the Fabry-Perot cavity as a result of the presence of the Au back mirror. Because the wavelength of the $\mathrm{Ar}^{+}$laser is nonresonant, the input laser light did not resonate in the cavity, which would have enhanced the PL further. The $4 \times$ enhancement at the resonances are clearly due to the combined effect of the enhancement of the PL by the cavity resonances with that of the input laser reflecting from the Au back mirror. That the PL dips have the same amplitude in both spectra is due to inhibition of the PL between the resonances.

\section{HYDROGENATED AMORPHOUS SILICON NITRIDE MICROCAVITY FABRICATED WITH AMMONIA}

The microcavity fabricated with $\mathrm{NH}_{3}$ had an $\mathrm{Al}$ back mirror and an $a-\mathrm{SiN}_{x}: \mathrm{H}$-air interface (or an $a-\mathrm{SiN}_{x}: \mathrm{H}-\mathrm{Al}$ partially transmitting) front mirror. First we coated the thin Si or quartz substrates with $100 \mathrm{~nm}$ of Al to fabricate the high-reflectance $\mathrm{Al}$ back mirror. Then we deposited a thin layer of $a-\mathrm{SiN}_{x}: \mathrm{H}$ onto the Al-coated substrates by PECVD. The flow rates of the PECVD gases were 180 SCCM for $2 \% \mathrm{SiH}_{4}$ in $\mathrm{N}_{2}$ and $10 \mathrm{SCCM}$ for $\mathrm{NH}_{3}$, the rf power was $10 \mathrm{~W}$, and the pressure of the deposition chamber was 1 Torr. The metric thickness $(L)$ of the $a$-SiN $x: \mathrm{H}$ layer grown with $\mathrm{NH}_{3}$ was $1000 \pm 100 \mathrm{~nm}$. Finally, the samples were annealed in a forming gas atmosphere at $600{ }^{\circ} \mathrm{C}$ for $10 \mathrm{~min}$. For the $\mathrm{Al}$ partially transmitting front mirror microcavities a thin (approximately 10-nm) layer of $\mathrm{Al}$ was deposited upon the annealed samples.

\section{A. Transmittance, Reflectance, and Absorbance of an} $a-S N_{x}: H$ Microcavity Fabricated with Ammonia

Figures 3(a), 3(b), and 3(c), respectively, show the measured and calculated transmittance, reflectance, and absorbance spectra of the $a-\mathrm{SiN}_{x}: \mathrm{H}$ microcavity fabricated with $\mathrm{NH}_{3}$. We obtained the absorbance spectrum by subtracting both the reflectance and the transmittance spec- 
tra from unity, i.e., $100 \%$. The calculations were performed with the absorbing Fabry-Perot microcavity model described above.

The theoretical fit to the experimental spectra is extremely good. The metric thickness of the $a-\mathrm{SiN}_{x}: \mathrm{H}$ grown with $\mathrm{NH}_{3}$ was $L=963 \mathrm{~nm}$ (which agrees well with the experimental thickness of $1000 \pm 100 \mathrm{~nm}$ ), and the refractive index was $n=1.6$. Both the transmittance (maxima) and the reflectance (minima) spectra showed Fabry-Perot resonances at wavelengths $\left(\lambda_{m}\right)$ that satisfied the microcavity resonance condition of Eq. (1). The mode numbers of these resonances ranged from $m$ $=7\left(\lambda_{7}=461 \mathrm{~nm}\right)$ to $m=4\left(\lambda_{4}=760 \mathrm{~nm}\right)$. The resonances had linewidths of $\Delta \lambda=40 \mathrm{~nm}$ and quality factors of $Q=10$. Compared with the absorption of the $a-\mathrm{SiN}_{x}: \mathrm{H}$ grown without $\mathrm{NH}_{3}$, the absorption of the $a-\mathrm{SiN}_{x}: \mathrm{H}$ grown with $\mathrm{NH}_{3}$ was an order of magnitude less. Consequently there was no loading of the resonances by the absorption.
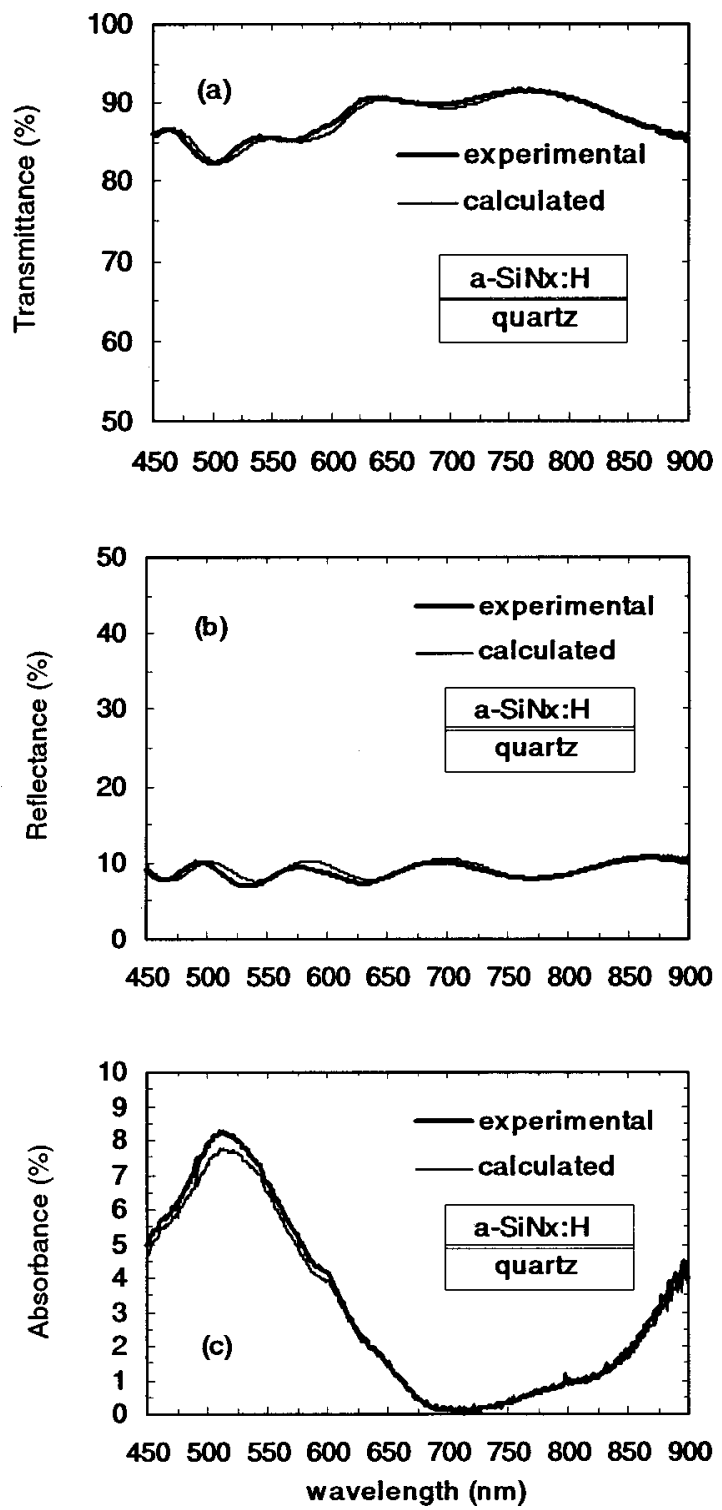

Fig. 3. Experimental and theoretical transmittance, reflectance, and absorbance spectra of an $a-\mathrm{SiN}_{x}: \mathrm{H}$ microcavity fabricated with $\mathrm{NH}_{3}$.
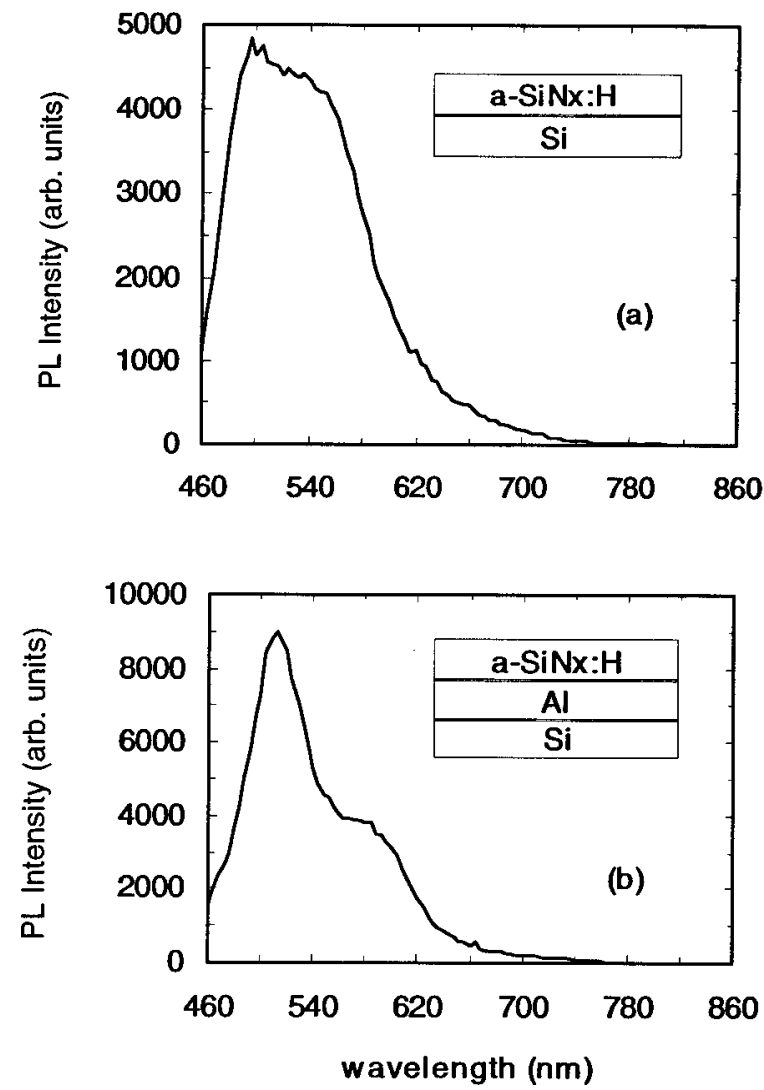

Fig. 4. PL spectra of the $a-\mathrm{SiN}_{x}: \mathrm{H}$ microcavity fabricated with $\mathrm{NH}_{3}$ (a) without and (b) with the $\mathrm{Al}$ mirror.

\section{B. Photoluminescence of $a-\mathrm{SiN}_{x}: \mathrm{H}$ Microcavity}

Fabricated with Ammonia

Figure 4(a) shows the PL spectrum of $a-\mathrm{SiN}_{x}: \mathrm{H}$ grown with $\mathrm{NH}_{3}$ upon $\mathrm{Si}$ together with that of the sample grown upon quartz, whose transmittance, reflectance, and absorbance spectra were shown in Fig. 3. The samples grown upon quartz were used for transmittance and reflectance measurements. The samples grown upon Si were used for PL measurements because $\mathrm{Al}$ adheres better to $\mathrm{Si}$ than to quartz. The PL intensity in Fig. 4(a) was modulated by the weak Fabry-Perot resonances. The mode numbers of these resonances ranged from $m=7\left(\lambda_{7}\right.$ $=488 \mathrm{~nm})$ to $m=6\left(\lambda_{6}=572 \mathrm{~nm}\right)$. PL was inhibited between these two resonances at $m=6.5\left(\lambda_{6.5}=524\right.$ $\mathrm{nm})$. The blue-green PL also had a broad linewidth (100 $\mathrm{nm}$ ), which, again demonstrates that $a-\mathrm{SiN}_{x}: \mathrm{H}$ has potential for use as a novel photonic gain medium. The Fabry-Perot resonances had experimental and theoretical linewidths of $\Delta \lambda=40 \mathrm{~nm}$ and quality factors of $Q$ $=10$. The metric thickness of the $a-\mathrm{SiN}_{x}: \mathrm{H}$ grown upon Si was $L=1103 \mathrm{~nm}$ (which agrees well with the experimental thickness of $1000 \pm 100 \mathrm{~nm}$ ), and the refractive index was $n=1.6$.

Figure 4(b) depicts the PL of the $a-\operatorname{SiN}_{x}: \mathrm{H}$ microcavity with the $\mathrm{Al}$ back mirror. Al was chosen instead of Au because $\mathrm{Al}$ has a higher reflectance in the blue-green part of the optical spectrum. The Fabry-Perot resonances had experimental and theoretical linewidths of $\Delta \lambda=60 \mathrm{~nm}$ and quality factors of $Q=8$. The metric thickness of the $a-\mathrm{SiN}_{x}: \mathrm{H}$ microcavity with the $\mathrm{Al}$ mirror was $L$ 
$=929 \mathrm{~nm}$ (which agrees well with the experimental thickness of $1000 \pm 100 \mathrm{~nm}$ ), and the refractive index was $n=1.6$. The PL was modulated by the strong Fabry-Perot resonances. The mode numbers of these resonances ranged from $m=6\left(\lambda_{6}=515 \mathrm{~nm}\right)$ to $m$ $=5\left(\lambda_{5}=603 \mathrm{~nm}\right)$.

The two PL spectra of Figs. 4(a) and 4(b) were obtained under the same experimental conditions. Comparing the spectra in Figs. 4(a) and 4(b) revealed that the PL of the microcavity with the $\mathrm{Al}$ mirror has three noteworthy features with respect to the PL of the $a-\mathrm{SiN}_{x}: \mathrm{H}: \quad$ (1) there is a $1.3 \times$ increase of the overall spectrum average (i.e., averaging out of the Fabry-Perot resonances), (2) there is a $2 \times$ enhancement of the PL peaks, and (3) the PL dips have similar amplitudes. The $1.3 \times$ increase is due to the round trip of the $\mathrm{Ar}^{+}$laser in the cavity that is due to the presence of the Al back mirror instead of a Si substrate, which only partially reflects the incident beam. Because the wavelength of the $\mathrm{Ar}^{+}$laser is nonresonant, the input laser light does not resonate in the cavity; such resonance would have enhanced the PL further. The $2 \times$ enhancement at the resonances is clearly due to the combined effect of the enhancement of the PL by the cavity resonances and that of the input laser reflecting from the $\mathrm{Al}$ back mirror. That the PL dips have the same amplitude in both spectra is due to the inhibition of PL between the resonances.

Figure 5(a) shows the PL of $a-\mathrm{SiN}_{x}: \mathrm{H}$ grown with $\mathrm{NH}_{3}$ [the same as that in Fig. 4(a)] divided by 15 to yield the same spectral average for both Figs. 5(a) and 5(b). Fig-
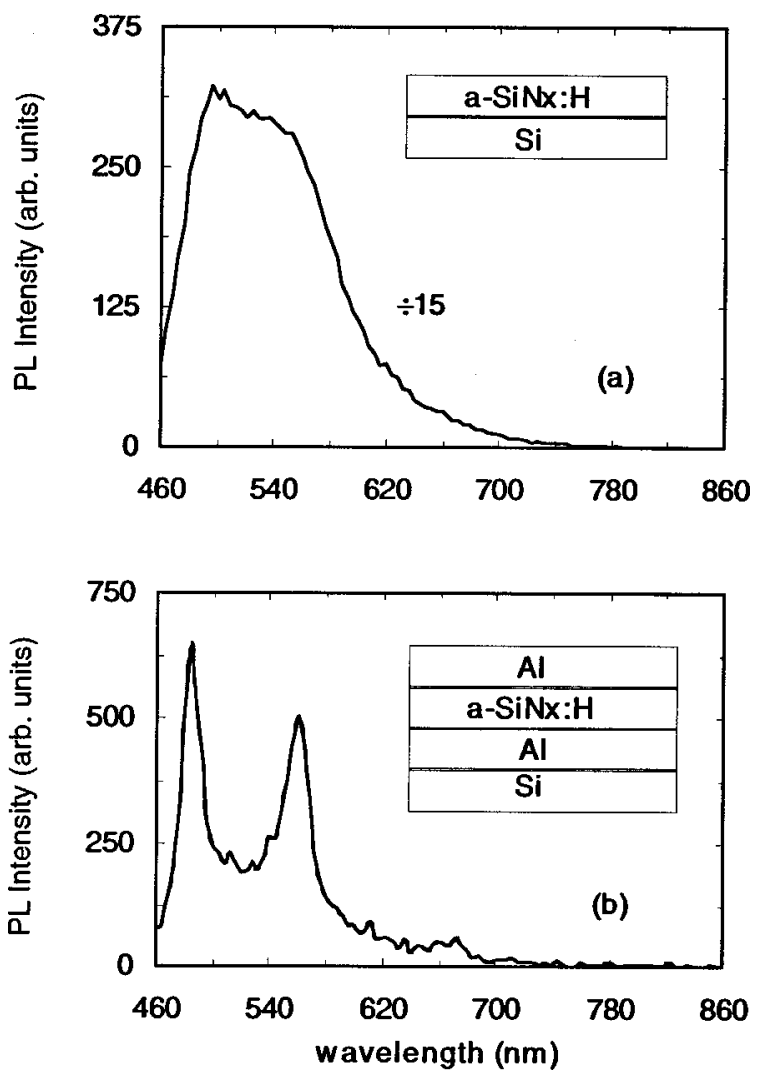

Fig. 5. PL spectra of the $a-\mathrm{SiN}_{x}: \mathrm{H}$ microcavity fabricated with $\mathrm{NH}_{3}$ (a) without the $\mathrm{Al}$ back mirror and (b) with the $\mathrm{Al}$ back and front mirrors. ure 5(b) depicts the PL of the $a-\mathrm{SiN}_{x}: \mathrm{H}$ microcavity with $\mathrm{Al}$ back and $\mathrm{Al}$ front partially transmitting mirrors. The Fabry-Perot resonances had experimental and theoretical linewidths of $\Delta \lambda=20 \mathrm{~nm}$ and quality factors of $Q=25$. The metric thickness of the $a-\mathrm{SiN}_{x}: \mathrm{H}$ microcavity with the $\mathrm{Al}$ back and partially transmitting front mirrors was $L=1072 \mathrm{~nm}$ (which agrees well with the experimental thickness of $1000 \pm 100 \mathrm{~nm}$ ), and the refractive index was $n=1.6$. The PL was modulated by strong Fabry-Perot resonances. The modes number of these resonances ranged from $m=7\left(\lambda_{7}=488 \mathrm{~nm}\right)$ to $m=5\left(\lambda_{5}=687 \mathrm{~nm}\right)$.

The PL spectrum of Fig. 5(b) was obtained under the same experimental conditions as for Fig. 5(a). Comparing the spectra in Figs. 5(a) and 5(b) reveals that the PL of the microcavity with the $\mathrm{Al}$ back and the partially transmitting $\mathrm{Al}$ front mirrors has three noteworthy features with respect to the PL of the $a-\mathrm{SiN}_{x}: \mathrm{H}$ : (1) the overall spectral averages are the same (i.e., averaging out of the Fabry-Perot resonances), (2) there is a $2 \times$ enhancement of the PL peaks, and (3) the PL dips have similar amplitude. The $2 \times$ enhancement at the resonances, while the overall average is the same, is clearly due to the enhancement of the PL by the cavity resonances. That the PL dips have the same amplitude in both spectra is due to the inhibition of the PL between the resonances.

\section{CONCLUSIONS}

In conclusion, Fabry-Perot microcavities with dielectric and metallic mirrors have been used for alteration of the $\mathrm{PL}$ in $a-\mathrm{SiN}_{x}: \mathrm{H}$ grown with and without $\mathrm{NH}_{3}$. The PL of the bulk $a-\operatorname{SiN}_{x}: \mathrm{H}$ is from the red to the near-infrared parts of the optical spectrum for the samples grown without $\mathrm{NH}_{3}$. However, the PL of the bulk $a-\mathrm{SiN}_{x}: \mathrm{H}$ is in the blue-green part of the optical spectrum for the samples grown with $\mathrm{NH}_{3}$. These complementary colors cover the entire visible spectrum, and one can imagine a color flatpanel display made of $a-\mathrm{SiN}_{x}: \mathrm{H}$ pixels. The broad PL spectrum of the $a-\mathrm{SiN}_{x}: \mathrm{H}$ also makes it a suitable source for wavelength-division multiplexing applications.

The PL of bulk $a-\mathrm{SiN}_{x}: \mathrm{H}$ is enhanced at, and inhibited between, the Fabry-Perot resonances of the microcavity. The enhancement and inhibition of the PL are understood as being due to the modified photon density of states of the microcavity. The linewidth of the PL is also narrower than the linewidth of the bulk $a-\mathrm{SiN}_{x}: \mathrm{H}$, again because of to the presence of the electromagnetic modes of the microcavity. The Fabry-Perot enhancement and inhibition of luminescence in $a-\mathrm{SiN}_{x}: \mathrm{H}$ open a variety of possibilities for optoelectronic applications such as color flat panel displays and RCE devices.

\section{ACKNOWLEDGMENTS}

We acknowledge partial support of this research by the Scientific and Technical Research Council of Turkey, grant TBAG-1368, and the International Center for Theoretical Physics, grant 95-500 RG/PHYS/AS. 
*Permanent address, Department of Physics, Bilkent University, Bilkent, Ankara 06533 Turkey.

\section{REFERENCES}

1. A. M. Agarwal, L. Liao, J. S. Foresi, M. R. Black, X. Duan, and L. C. Kimerling, "Low-loss polycrystalline silicon waveguides for silicon photonics," J. Appl. Phys. 80, 6120 (1996).

2. C. Gorecki, F. Chollet, E. Bonnonte, and H. Kawakatsu, "Silicon-based integrated interferometer with phase modulation driven by surface acoustic waves," Opt. Lett. 22, 1784 (1997).

3. D. J. Lockwood, "Optical properties of porous silicon," Solid State Commun. 92, 101 (1994).

4. T. Canham, "Silicon quantum wire array fabrication by electrochemical and chemical dissolution of wafers," Appl. Phys. Lett. 57, 1046 (1990).

5. P. C. Sercel, D. Kwon, T. Vilbrandt, W. Yang, J. Hautala, J. D. Cohen, and H. Lee, "Visible electroluminescence from porous silicon/hydrogenated amorphous silicon pnheterojunction devices," Appl. Phys. Lett. 68, 684 (1996).

6. J. Rarity and C. Weisbuch, eds., Microcavities and Photonic Bandgaps: Physics and Applications (Kluwer, Dordrecht, The Netherlands, 1996).

7. E. M. Purcell, "Spontaneous emission probabilities at radio frequencies," Phys. Rev. 69, 681 (1946).

8. F. De Martini, G. Innocenti, G. R. Jacobowitz, and P. Mataloni, "Anomalous spontaneous emission time in a microscopic optical cavity," Phys. Rev. Lett. 59, 2955 (1987).

9. H. Yokoyama and S. D. Brorson, "Rate equation analysis of microcavity lasers," J. Appl. Phys. 66, 4801 (1989).

10. M. S. Ünlü and S. Strite, "Resonant cavity enhanced photonic devices," J. Appl. Phys. 78, 607 (1995).

11. E. F. Schubert, Y.-H. Wang, A. Y. Cho, I. W. Tu, and G. J. Zydzik, "Resonant cavity light emitting diode," Appl. Phys. Lett. 60, 921 (1992).
12. H. Yokoyama, K. Nishi, T. Anan, H. Yamada, S. D. Brorson, and E. P. Ippen, "Enhanced spontaneous emission from GaAs quantum wells in monolithic microcavities," Appl. Phys. Lett. 57, 2814 (1990).

13. S. L. McCall, A. F. J. Levi, R. E. Slusher, S. J. Pearton, and R. A. Logan, "Whispering gallery mode microdisk lasers," Appl. Phys. Lett. 60, 289 (1992).

14. J. P. Zhang, D. Y. Chu, S. L. Wu, S. T. Ho, W. G. Bi, C. W. Tu, and R. C. Tiberio, "Photonic wire lasers," Phys. Rev. Lett. 75, 2678 (1995).

15. L. Pavesi, R. Guardini, and C. Mazolleni, "Porous silicon resonant cavity light emitting diodes," Solid State Commun. 97, 1051 (1996).

16. B. T. Sullivan, D. J. Lockwood, H. J. Labbe, and Z.-H. Lu, "Photoluminescence in amorphous $\mathrm{Si} / \mathrm{SiO}_{x}$ superlattices fabricated by magnetron sputtering," Appl. Phys. Lett. 69, 3149 (1996).

17. F. N. Timofeev, A. Aydinli, R. Ellialtioglu, K. Türkoglu, M. Güre, V. N. Mikhailov, and O. A. Lavrova, "Visible photoluminescence from $\mathrm{SiO}_{x}$ films grown by low temperature plasma enhanced chemical vapor deposition," Solid State Commun. 95, 443 (1995).

18. A. Serpengüzel, A. Aydinli, and A. Bek, "Enhancement and inhibition of photoluminescence in hydrogenated amorphous silicon nitride microcavities," Opt. Express 1, 108 (1997).

19. A. Aydinli, A. Serpengüzel, and D. Vardar, "Visible photoluminescence from low temperature deposited hydrogenated amorphous silicon nitride," Solid State Commun. 98, 273 (1996).

20. M. J. Estes and G. Moddel, "A model of size dependent photoluminescence in amorphous silicon nanostructures: comparison with observations of porous silicon," Appl. Phys. Lett. 68, 1814 (1996).

21. M. H. Brodsky, "Quantum well model of the hydrogenated amorphous silicon," Solid State Commun. 36, 55 (1980).

22. R. Fisher, in Amorphous Semiconductors, M. H. Brodsky, ed. (Springer-Verlag, Berlin, 1985), pp. 159-187. 